\title{
L'intervisibilità. Analisi del paesaggio nella chora della colonia greca di Himera
}

\author{
Maria Assunta Papa \\ Laboratorio di Topografia Antica, Università di Palermo \\ email: marypapa@virgilio.it
}

\begin{abstract}
This study focuses on the analysis of the landscape in the territory of the colony of Himera. In particular, this work is inspired by a detailed examination conducted on the basis of parametric modelling, which has helped us to understand what factors (pedology, altimetry, geology, supply basins, etc.) had an effect in the settlement choices of the Imerese territory during the Colonial Age. We analysed the role intervisibility had between sites in the organisation of the landscape. By avoiding a deterministic logic, we intersected the data from previous surveys on the development of predictive models with the archaeological data, formulating hypotheses on the role that the main settlements in the area had on the control and economic management of the territory.
\end{abstract}

Keywords: Chora, Settlement parameters, Landscape, intervisibility, GIS

\section{Testo}

Lo studio che proponiamo è focalizzato sull'analisi del paesaggio nel territorio della colonia greca di Himera in Sicilia. Questo lavoro prende spunto da un esame dettagliato condotto sulla base della modellistica parametrica, che ci ha aiutato a comprendere quali fattori (pedologia, altimetria, geologia, bacini di approvvigionamento, toponomastica etc.) abbiano influito nelle scelte insediative del territorio imerese in età coloniale.
L'attività di ricerca si è focalizzata sul monitoraggio delle attività umane nel territorio Imerese, al fine di predisporre di modelli idonei alla comprensione delle dinamiche insediamentali, ai caratteri del popolamento e dello sfruttamento delle risorse.

L'informazione geografica, geomorfologica e storica ricavabile dai dati raccolti nelle prospezioni della chora imerese (Burgio, 2002; Lauro, 2010; Vassallo, 1990) condotta dal Dipartimento di Beni Culturali dell'Università di Palermo ci è sembrato potesse rappresentare, grazie al dettaglio e alla metodologia della ricerca portata avanti in questi anni, una base esemplare per spiegare, analizzare ed interpretare la storia del popolamento antico nei territori delle antiche colonie greche.

In questa sede non tratteremo dei risultati ottenuti dalla nostra ricerca per quanto riguarda la costruzione dei modelli predittivi realizzati attraverso l'analisi dei differenti parametri utilizzati (Papa, 2012; 2013), ma analizzeremo il ruolo dell'intervisibilità tra i siti nell'organizzazione del paesaggio, cercando di evitare logiche di tipo deterministico.

I presupposti teorici e metodologici alla base della ricerca che tratteremo in questo paragrafo sono rappresentati dalle più recenti esperienze maturate in ambito europeo attorno alla Settlement Archaeology, in particolare da quelle orientate su scala regionale (Attema et al, 2002; Francovich, Patterson \& Barker, 2000). Tali ricerche sono state generalmente sviluppate utilizzando dati provenienti da indagini di superficie e presentano un approccio 


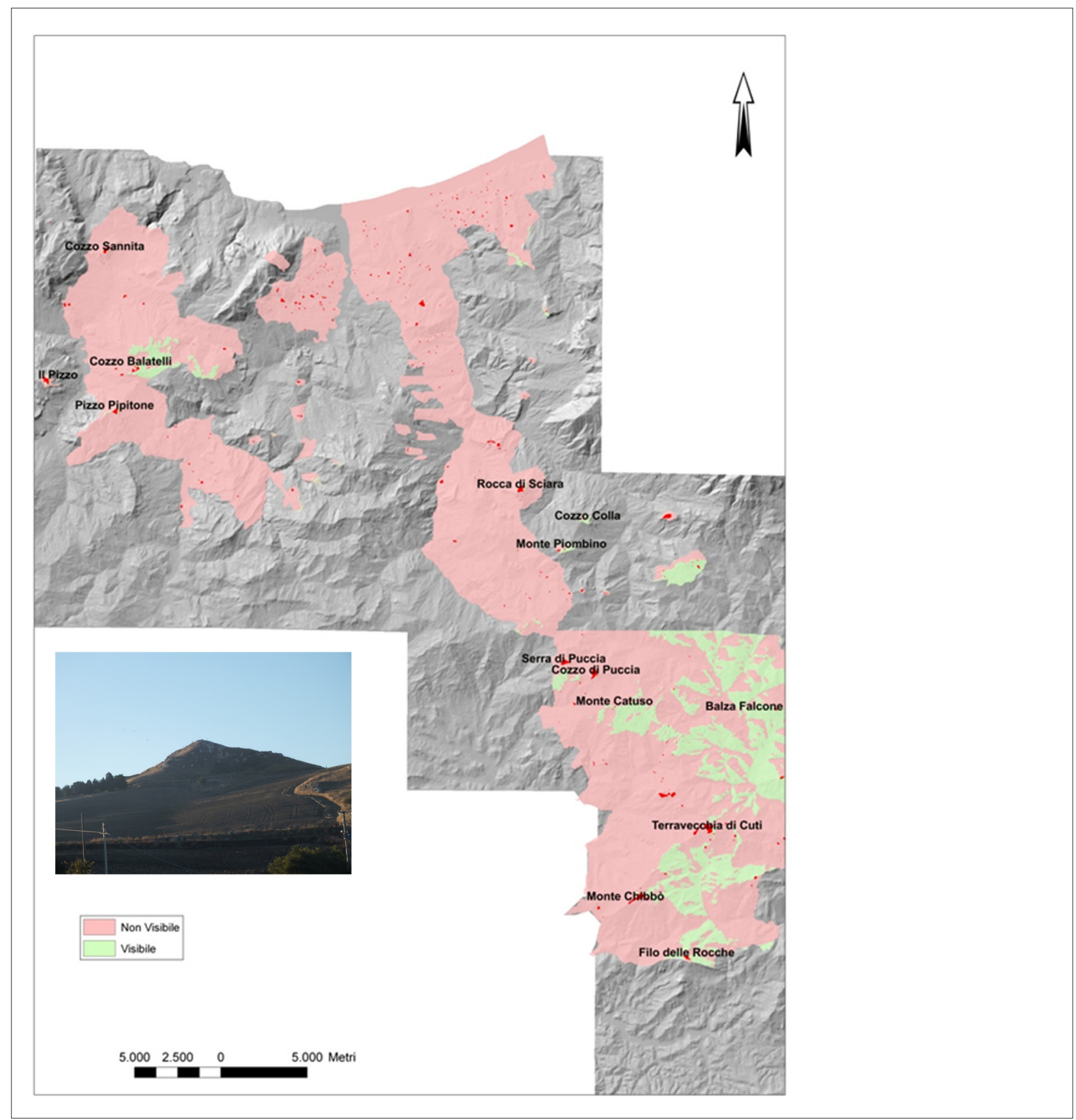

Fig. 1. Analisi di Intervisibilità da Terravecchia di Cuti.

metodologico idoneo per gestire questo tipo d'informazioni; inoltre, i risultati di tali applicazioni, per la natura stessa dei dati utilizzati, vengono interpretati soprattutto in un'ottica predittiva. L'obiettivo che ci siamo proposti è quello di utilizzare i risultati delle analisi spaziali, in particolare delle viewshed, per formulare nuove ipotesi di lettura mirate a valorizzare le dinamiche endogene di occupazione e di popolamento della regione. Nella necessità di comprendere il grado di aggregazione e di articolazione dei sistemi di insediamento e di individuare le trasformazioni diacroniche e sincroniche del territorio, l'indagine è stata impostata su un livello di analisi che considera il singolo insediamento in rapporto al territorio circostante e al paesaggio antropico che 
doveva caratterizzarlo in età greca (Clarke, 1977).

L'insediamento, che costituisce, nelle analisi di intervisibilità, l'unità primaria di osservazione, viene inteso come un luogo d'incontro di forme sociali, economiche e spirituali di una comunità. Le viewshed analyses consentono di arrivare alla simulazione complessa delle relazioni tra morfologia del paesaggio e sistemi insediativi. Questi applicativi, offerti dal software GIS Arc Map, elaborando i dati da noi prescelti, consentono di determinare il campo di osservazione rispetto all'orizzonte visivo di un osservatore mostrando le relazioni tra i diversi insediamenti e l'ambiente circostante. Queste analisi si basano sul concetto di "will to visibility" esposto nello studio di D. Wheatley e M. Gillings (2002; 201-16). L'aspetto visivo di un luogo viene analizzato nella nostra ricerca in quanto è valutato da noi come un fattore significativo nella selezione di luoghi adatti per l'impianto di un insediamento, di un luogo di culto, di un monumento di particolare rilievo per la comunità o di altre attività produttive (fattorie, insediamenti rurali, fornaci, ecc.). Inoltre, ovviamente, la determinazione della posizione di un insediamento non può non considerarsi in rapporto alle esigenze di difesa e controllo del territorio.

Un'analisi di intervisibilità completa della nostra area studio, valutando ogni singolo insediamento in rapporto agli altri, avrebbe rallentato molto i tempi di realizzazione del modello predittivo all'interno del nostro studio a discapito della ricerca metodologica che risulta essere il fine principale di questo lavoro. Si è pertanto stabilito di analizzare l'intervisibilità del territorio rispetto a dieci importanti insediamenti, scelti sulla base delle informazioni bibliografiche e storico-archeologiche che avevamo a nostra disposizione.

La scelta ha portato all'elaborazione delle analisi di intervisibilità su quattro grandi insediamenti rurali, su quattro insediamenti di altura identificati come grandi centri abitati e su altri tre insediamenti interpretati come santuari. La scelta non è stata semplice e ha influito molto su di essa la tipologia degli insediamenti, i materiali rinvenuti in essi, la loro estensione e la loro importanza bibliografica.
Gli insediamenti rurali prescelti sono posti in comprensori territoriali differenti quali la Valle del San Leonardo (UT 257 - Contrada Ciacca: Lauro, 2010: 81-83); il territorio di dell'alta valle dell'Imera (UT 182 - Contrada Ciampanella: Burgio, 2002: 130-32); la bassa Valle del Torto (UT 272 - Casa Franco: Cucco, 1999-2000: 57-60, 98-99) e l'area del Torrente Caltavuturo (UT 233 Contrada Stripparia: Fiorani, 2004-2005: 295-303). La scelta degli insediamenti di altura è caduta sui grandi insediamenti di Cozzo Puccia (UT 54: Burgio, 2002: 51-52; Belvedere et al 2002, 22325), del Pizzo di Ciminna (UT 245: Di Leonardo, 2007: 65-67; Lauro, 2010: 81-83; Vassallo, 1996); di Rocca di Sciara (UT 219: Fiorani, 2004-2005: 10713) e di Terravecchia di Cuti (UT 54: Belvedere, 2010; Epifanio, 1993; 1980; Vassallo, 1990: 48-63). Per quanto riguarda le aree santuariali (Belvedere, 2010) sono stati presi in considerazione i siti di Terravecchia di Cuti (UT 9: Epifanio, 1993; 1980; Vassallo, 1990: 48-63); di Serra di Puccia (UT 156: Burgio, 2002: 47-51; Belvedere et al 2002, 215-19); e di Cozzo Tutusino (UT 82: Belvedere et al 2002: 164-74).

Dal punto di vista tecnico le viewshed analyses si applicano su un modello digitale del terreno (DTM) e sfruttano algoritmi GIS per calcolare la porzione di territorio percepibile da uno o più osservatori collocati in un determinato punto rispetto alle direzioni di osservazione. Per calibrare l'algoritmo GIS è stata considerata un'altezza dell'osservatore di $1.70 \mathrm{~m}$ e un raggio di osservazione tra i 15 e 20 $\mathrm{km}$, che corrisponde all'ampiezza del campo visivo in condizioni metereologiche ottimali (Wheatley \& Gillings, 2002: 210-12). L'analisi di viewshed consiste nel calcolare il bacino visuale rispetto alla posizione e all'orizzonte visivo di un osservatore. Grazie a questa funzione abbiamo ottenuto delle immagini raster, che conservano le stesse proprietà del DEM di partenza lrisoluzione pixel $50 \mathrm{~m}$ per $50 \mathrm{~m}$ ), ma con la seguente caratteristica aggiuntiva: ogni cella che ricade lungo una line of sight interrotta è classificata come visible lvalore pari a 1), mentre quelle attraversate da una line of sight intercettata dai valori di elevazione delle altre celle è classificata come not visible lvalore 


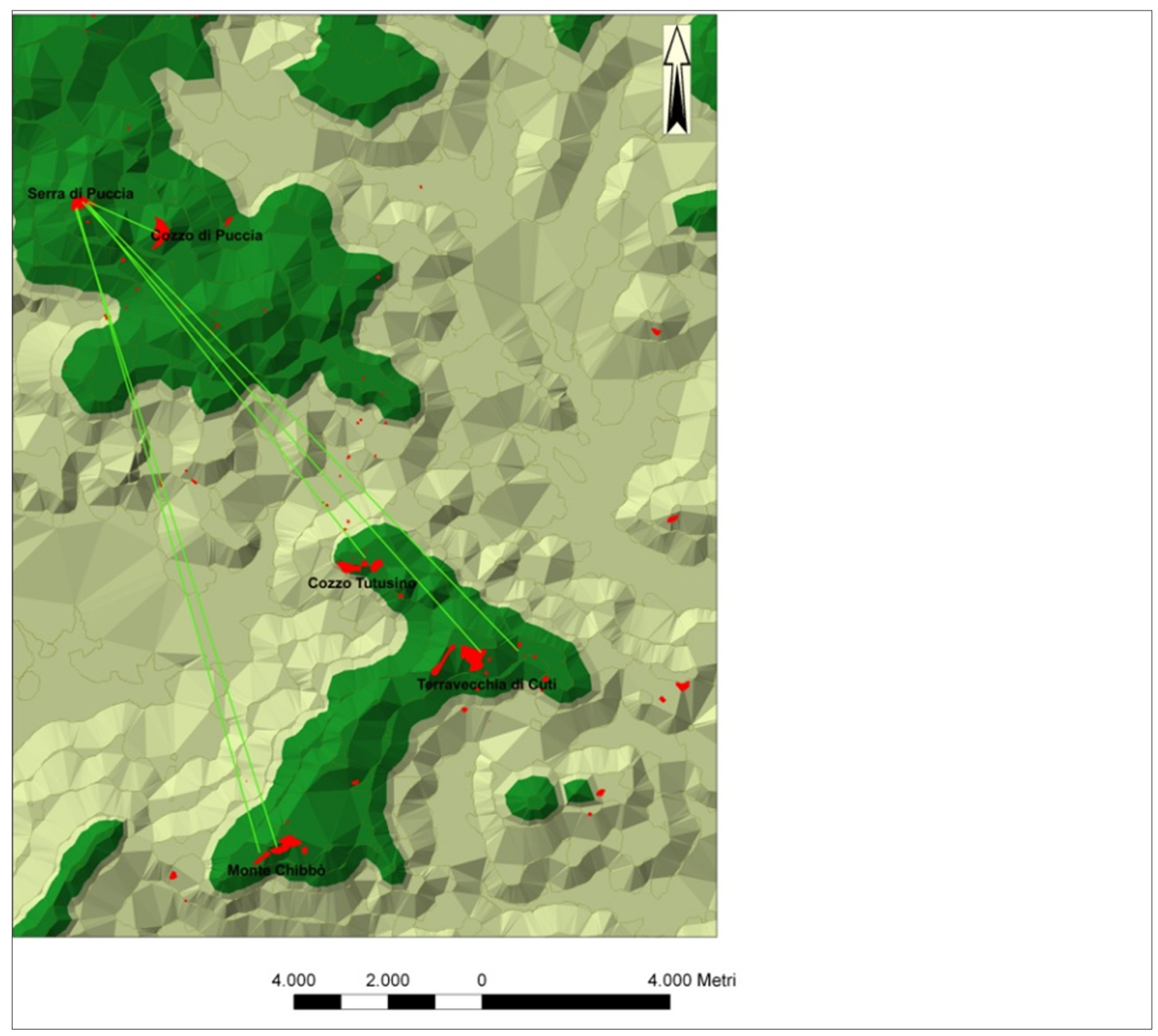

Fig. 2. Alta valle dell'Imera. Intervisibilità tra santuari.

pari a 0). La viewshed analysis dunque consente di ottenere un'immagine raster in cui il valore di ogni cella può essere fondamentalmente 0 o 1.

Inizieremo a trattare delle analisi di intervisibilità effettuate su quattro dei principali siti di altura della nostra area campione. Le UT prescelte riguardano i tre principali grandi abitati di altura disposti in aree strategiche nel territorio della nostra ricerca a quote che superano gli 800 m slm IUT 54 - Cozzo Puccia-1034 m slm; UT 245 - Il Pizzo di Ciminna-810 m slm; UT 219 - Rocca di
Sciara-1080 m slm, UT 5 - Terravecchia di Cuti$961 \mathrm{~m} \mathrm{~s}$ sm). Le analisi d'intervisibilità ottenute dalla scelta di queste UT ha portato risultati eccezionali, dimostrando come questi grandi centri non solo controllavano il territorio circostante ma creavano delle vere e proprie reti visive che dovevano avere un forte significato simbolico. Infatti, riteniamo che se la necessità degli imeresi fosse solamente stata un'esigenza di controllo territoriale e di difesa dell'area posta sotto il proprio dominio, questi insediamenti di altura non sarebbero stati così grandi, ma avrebbero potuto ben essere sostituiti da piccole aree di frammenti che ci potevano far ipotizzare la presenza di punti di vedetta. Dunque per gli antichi Greci il controllo 
territoriale era affidato a grandi insediamenti allo stesso tempo difendibili ma ben individuabili all'interno del comprensorio.

Ad esempio il viewshed ricavato sulla base dell'insediamento di Terravecchia di Cuti (fig. 1) registra come visibili UT 31, di cui alcune molto rilevanti come Cozzo Sannita, Cozzo Balatelli, Il Pizzo di Ciminna, Pizzo Pipitone, Rocca di Sciara, Cozzo di Puccia, Serra di Puccia, Balza Falcone, ecc.

La seconda analisi di intervisibilità è stata condotta usando come base l'UT 54 ovvero l'area dell'abitato di Cozzo di Puccia posto all'estremità orientale del massiccio lungo il quale corre lo spartiacque tra Imera e Platani. Questo comprensorio è interessato da un complesso di UT (UT $6,53,156,160,168$ ) che dimostrano una forte antropizzazione del paesaggio. Le tipologie insediamentali sono differenti (si riconoscono degli insediamenti rurali, un'area di necropoli e un santuario) ma le UT dovevano sicuramente avere stretti rapporti tra loro come dimostrano sia la ricostruzione viaria che l'analisi d'intervisibilità elaborata. Cozzo di Puccia rappresentava dunque il nucleo abitativo principale di questo interessante piccolo comprensorio ma doveva essere un centro indigeno ricco attivo sin da età arcaico-classica e aperto ai contatti culturali sia con i principali centri indigeni siciliani (sono attestati materiali del tipo Sant'Angelo Muxaro-Polizzellol sia con la costa (dai dati di ricognizione sembra siano attestati anche materiali d'importazione corinzi).

Esaminando la valle del San Leonardo ci è sembrato opportuno usare come punto di osservazione il Pizzo di Ciminna. Il Pizzo, rilevante centro indigeno sin da età arcaica, si erge su un rilievo di forma piramidale, costituito da un affioramento di gessi che dista circa $2 \mathrm{~km}$ dalla riva occidentale del San Leonardo. II Pizzo rappresenta un fondamentale punto di controllo delle vie di penetrazione interne alla Sicilia e si può ritenere che esso faccia parte di un sistema di roccaforti che controllavano e dominavano il paesaggio in età greca. La funzione di controllo territoriale doveva avere una particolare valenza per la roccaforte di Ciminna posta al confine tra i territori greci e quelli punici rafforzando l'idea di un insediamento nato in un luogo interessante dal punto di vista strategico ma ben difeso naturalmente. Analizzando la ricostruzione della viabilità antica, infatti sulla base dei nostri studi sembrava che l'insediamento doveva essere collegato alla costa e ad altri importanti centri interni, in rapporto alle potenzialità cerealicole dell'asse fluviale San Leonardo-Mendola-Sosio lil territorio della Valle del S. Leonardo si presenta marnoso e fertile e idoneo anche all'impianto di ulivetil si può credere che il Pizzo fosse il centro di riferimento per un ampio comprensorio interessato da una fiorente attività agricolocommerciale. Dal Pizzo di Ciminna sono visibili 46 UT, 16 di queste fanno parte della vallata del San Leonardo mentre molte delle altre, alla luce anche delle analisi di intervisibilità già commentate, possiamo affermare che siano punti strategici di una rete visiva che interessava tutta la chora imerese. Tra questi principali insediamenti ricordiamo: Cozzo Sannita, Pizzo Pipitone, Monte Chibbò, Monte Presepio, Rocca di Sciara, Serra di Puccia, Terravecchia di Cuti, etc..

Si potrebbe anche sottolineare per il Pizzo di Ciminna, alla luce delle notizie degli scavi di un complesso sacro dedicato alle divinità ctonie Demetra e Kore (Curti, 2003), che le analisi di intervisibilità colgono un rapporto tra il sito e altri santuari importanti del territorio imerese quali Serra di Puccia e Cozzo Tutusino. Non sembra meno importante anche la lettura di intervisibilità tra il Pizzo e centri rurali minori della Valle del Torto e della Valle dell'Imera che probabilmente dovevano essere collegati per ragioni economicosociali al principale centro della vallata del S. Leonardo.

L'ultimo insediamento di altura (Rocca di Sciaral di cui analizzeremo il viewshed è posto al limite del comprensorio piano-collinare della Valle occidentale dell'Imera Settentrionale in cui esso rappresenta il più elevato promontorio a controllo del territorio ma soprattutto è il nodo visivo tra l'insediamento di Imera e le principali roccaforti dell'entroterra (Cozzo di Puccia, Monte Catuso, Monte Chibbò, Terravecchia di Cuti). 
Ovviamente l'analisi d'intervisibilità dell'UT 219 evidenzia un legame particolare con le Vallate del Torto e dell'Imera e controlla numerose UT interpretabili come insediamenti rurali di una certa importanza. Infine dalla Rocca di Sciara sono visibili anche altre roccaforti, quelle del S.Leonardo (Cozzo Balatelle, Cozzo Cannita, Cozzo Pipitone). Da Rocca di Sciara si contano almeno 123 insediamenti visibili e ciò ci fa ipotizzare un ruolo di vero e proprio nodo a controllo del territorio greco.

Analizzando i materiali rinvenuti a Rocca di Sciara essi sono databili in età coloniale e l'insediamento non sembra avere forti influenze di matrice indigena, ciò ci fa ipotizzare la nascita di questo sito proprio in coincidenza della presa di potere del centro di Himera nel territorio. Rocca di Sciara nasce in un luogo eccezionalmente strategico e mi sembra interessante rilevare il fatto che sia l'unico dei quattro insediamenti analizzati che riesca a vedere direttamente la sua polis di riferimento.

Passando all'analisi dell'intervisibilità tra tre dei santuari individuati nell'alta valle dell'Imera i risultati non sono altrettanto evidenti. I siti scelti per questo studio possono essere visibili tra loro ma l'“observer point" deve essere di almeno $2.30 \mathrm{~m}$ e inoltre la scelta del posizionamento del punto di osservazione all'interno dell'area degli insediamenti non è stata semplice. Si può dunque affermare che i santuari non dovevano avere un forte impatto visivo immediato e che probabilmente erano protetti dall'ambiente circostante anche se però anche per questi insediamenti il rapporto visivo con le principali roccaforti a controllo del territorio era strategicamente assicurato.

L'UT 82 di Cozzo Tutusino, l'UT 156 di Serra di Puccia e l'UT 9 di Terravecchia di Cuti rappresentano bibliograficamente i tre principali santuari del territorio dell'alta valle dell'Imera (fig. 2). L'UT 9 è chiaramente in una posizione protetta e controllata da piccoli insediamenti satelliti anche se però rimane ben visibile per alcuni dei centri più interni come Monte Chibbò, Filo delle Rocche e Balza Falcone, abitati fortificati che probabilmente vedevano l'UT 9 come un centro di culto di riferimento. Più o meno lo stesso discorso si potrebbe affrontare per il santuario relativo al comprensorio di Cozzo Tutusino. Infatti l'UT 82 non gode di una intervisibilità eccellente e riesce a intercettare il posizionamento di solamente altre 15 UT Inella maggior parte dei casi insediamenti rurali). L'intervisibilità tra gli stessi santuari però è ancora presente.

Analizzando l'intervisibilità dell'UT 156 lda Serra di Puccia vengono intercettate visivamente solo 13 UT) si può notare come il territorio posto sotto il suo controllo visivo abbia un raggio poco più ristretto rispetto i due santuari UT 9 e UT 82; ma mi sembra opportuno sottolineare che da Serra di Puccia si riescono a intravedere sia il santuario di Cozzo Tutusino che il santuario di Terravecchia.

Inoltre si deve evidenziare che sia i grandi centri che le principali roccaforti (Monte Chibbò, Polizzi Generosa, Rocca di Sciara, Terravecchia) sono tutte collegate in rete visiva col santuario. Ricordiamo anche che l'intervisibilità ottenuta usando come punto di osservazione il Pizzo di Ciminna riusciva a intercettare il santuario di Serra di Puccia.

Si può dedurre che sia per la sua estensione (circa $6000 \mathrm{mq}$ ) che per la sua posizione il santuario di Serra di Puccia dovesse avere un ruolo principale per il territorio dell'alta valle dell'Imera. L'UT 156 infatti nonostante appartenga al comprensorio sociale composto anche dall'abitato-roccaforte di Cozzo di Puccia necessita di grandi spazi suoi. La sua posizione sebbene ad alta quota (1052 $\mathrm{m}$ slm) risulta interna e arroccata garantendogli però la protezione visiva dei grandi centri del territorio Itra i quali Cozzo di Puccia che doveva avere un legame strettissimo con il santuario). Allo stesso tempo la sua estensione e la sua rete di visibilità collegata agli altri santuari doveva assicurare a questo santuario una fiorente economia e probabilmente un alto valore simbolico di luogo di culto egemone posto in una posizione sicura e protetta.

Esaminando la zona costiera e aree a quote più basse sono state realizzate cinque mappe 


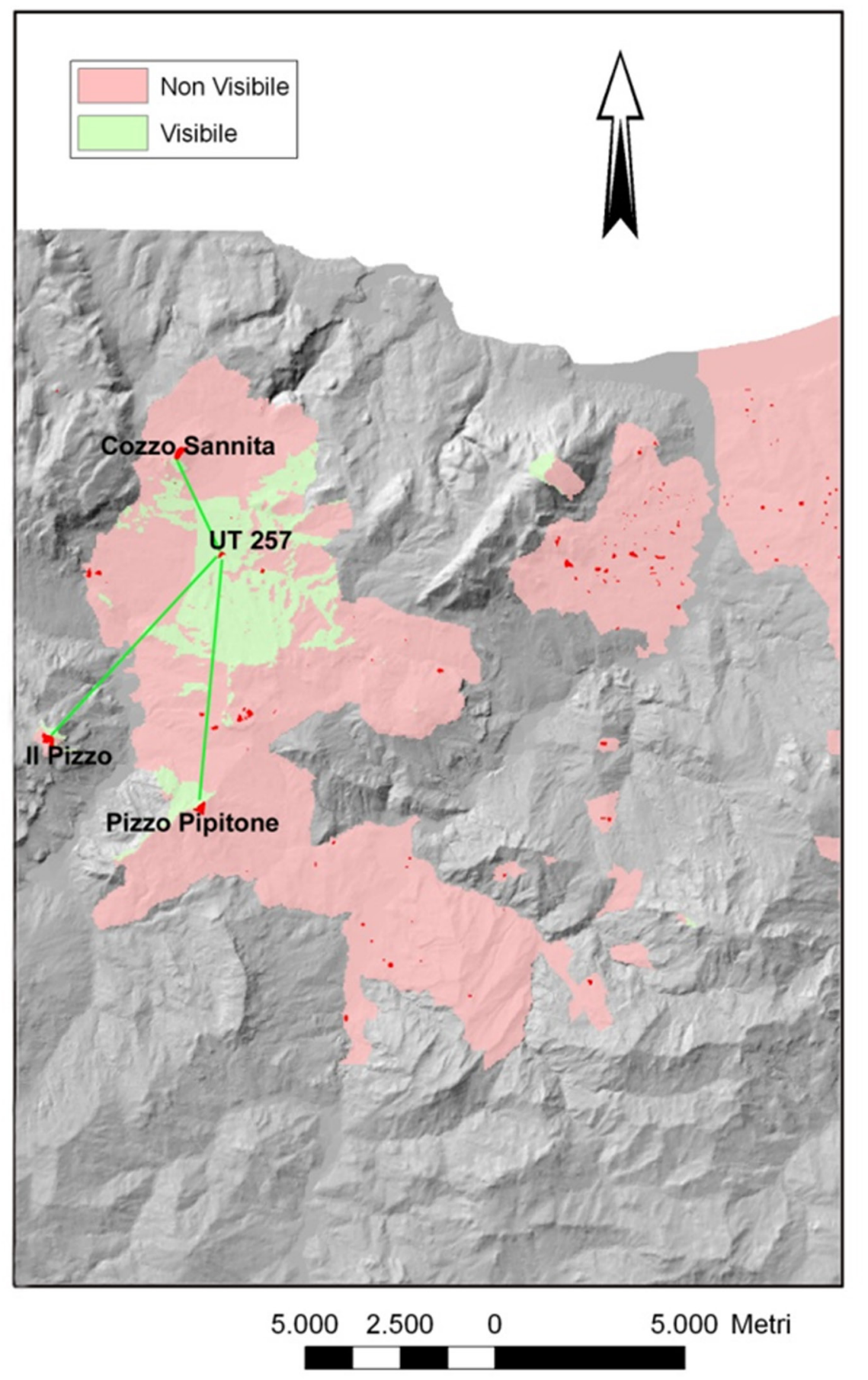

Fig. 3. Analisi di Intervisibilità dall'UT 257 (insediamento rurale della Valle del San Leonardo).

d'intervisibilità usando come punti di osservazione insediamenti rurali (UT 182, UT 233, UT 257, UT 272) piuttosto grandi e con una lunga continuità di vita (età arcaica-età ellenistica) e la polis di Himera.

I quattro insediamenti rurali hanno visibilità ridotte e il loro raggio visivo è molto legato ai comprensori a cui appartengono. Infatti ognuno di questi insediamenti mantiene un rapporto visuale con almeno tre insediamenti roccaforte o santuari posti in punti nodali: l'UT 182 riesce a intercettare il centro di Tutusino, Terravecchia di Cuti e Monte Catuso; l'UT 233 si vede con Rocca di Sciara; Monte Piombino e Polizzi Generosa; l'UT 


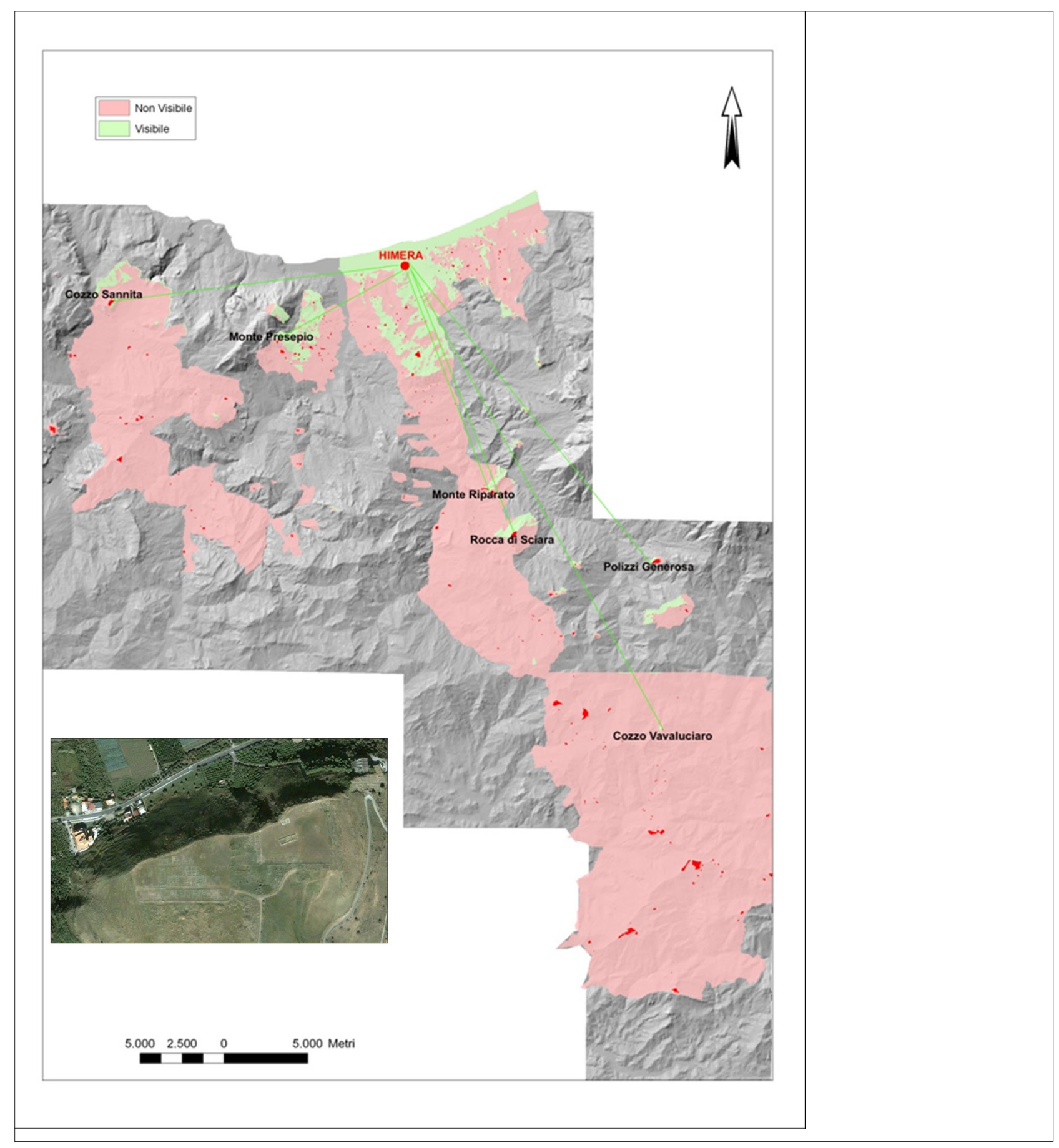

Fig. 4. Himera. Analisi di Intervisibilità.

257 scorge Cozzo Sannita, Pizzo Pipitone e Il Pizzo di Ciminna e l'UT 272 doveva avere nel suo raggio visivo Himera, Rocca di Sciara e Monte Presepio (fig. 3).

Il nostro ultimo viewshed realizzato sulla nostra area campione è stato costruito sulla base del posizionamento della città di Himera (fig. 4). Questa analisi ci ha fatto comprendere come le nostre idee riguardo a una rete visiva di insediamenti non fossero semplici astrazioni. Dall'osservazione di quest'ultimo elaborato e dal confronto con le mappe già esaminate abbiamo potuto dedurre come Himera, la grande città coloniale posta sulla costa, avesse un controllo 


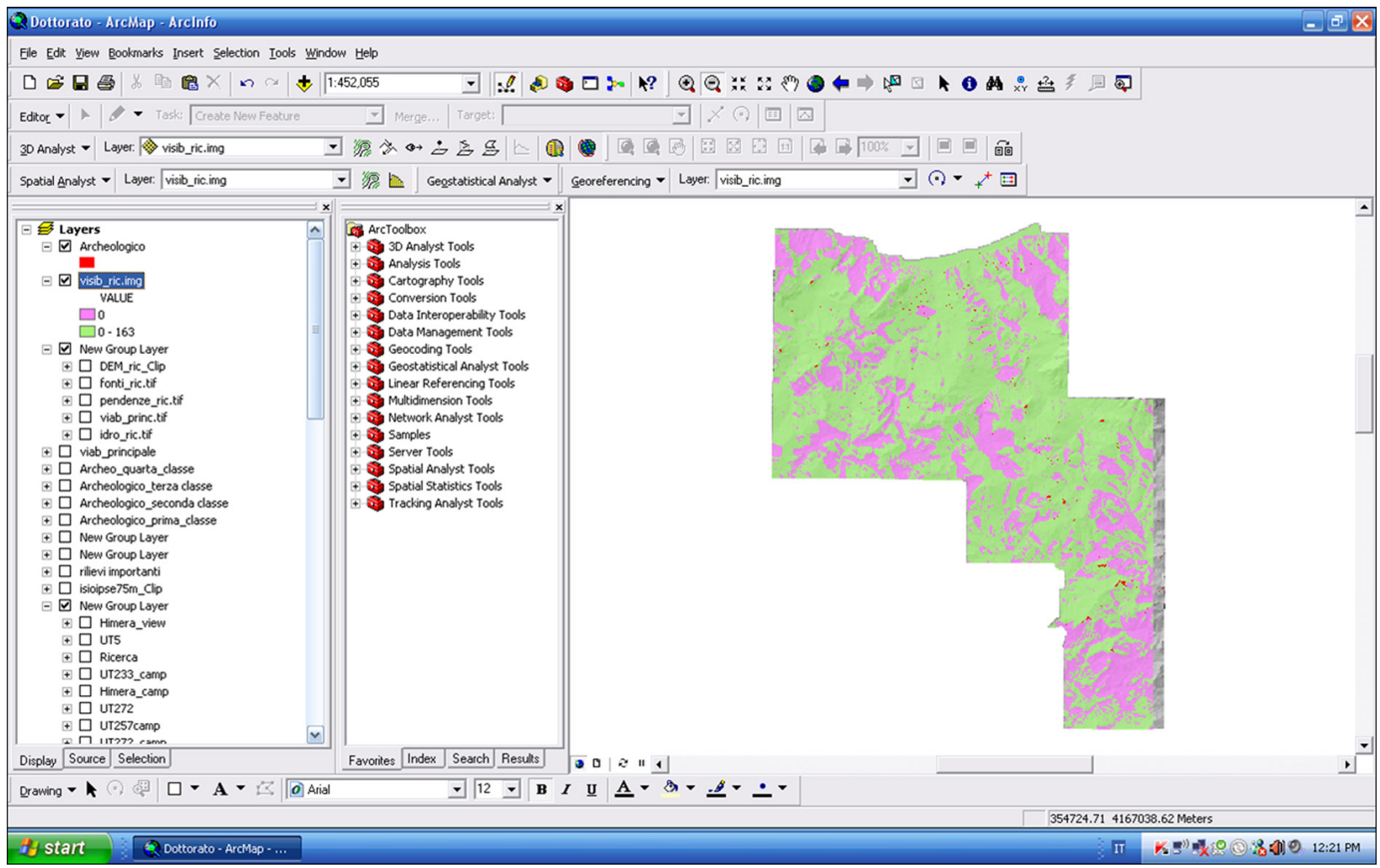

Fig. 5. File raster inserito nella costruzione dei modelli predittivi in ambiente GIS.

diretto sui territori limitrofi dove insistevano piccole fattorie, e un controllo politico-economico su tutta l'area della nostra ricerca assicurato da grandi roccaforti poste in luoghi strategicamente rilevanti. Rocca di Sciara, Monte Presepio, Polizzi Generosa e Cozzo Sannita erano i centri fortificati che principalmente dovevano garantire il "dialogo visivo" con le roccaforti più interne quali Il Pizzo di Ciminna; Pizzo Pipitone; Cozzo di Puccia; Monte Piombino; Terravecchia di Cuti; Monte Chibbò. Le regioni più interne erano interessate da insediamenti rurali che sfruttavano il potenziale agricolo del territorio mantenendo forti rapporti con queste roccaforti come dimostrano sia l'analisi dei collegamenti viari sia lo studio dell'intervisibilità.

Anche i santuari erano collegati ai principali nodi difensivi del territorio ma posti in aree più protette e riparate erano tutelati dai centribaluardi che li circondavano. I santuari comunque, pur essendo collocati a quote poco più basse e in luoghi geomorfologicamente più appartati rispetto alle cittadelle fortificate, mantengono fra loro rapporti di visibilità o quantomeno dialogano attraverso i centri roccaforte.

Alla luce di queste osservazioni riteniamo che l'analisi di intervisibilità riesca a cogliere una distribuzione insediamentale nel territorio imerese che doveva avere logiche simboliche, strategiche, difensive ed economiche ben articolate.

I risultati ottenuti si sono dimostrati molto significativi e con capacità predittive elevate: si è scelto dunque infine di effettuare una nuova analisi d'intervisibilità da applicare a tutta l'area della nostra ricerca utilizzando come punti di osservazione i principali nodi strategici che possiamo ipotizzare potessero costituire delle piazzeforti nel territorio dell'antica colonia (fig. 5). Oltre alla stessa colonia abbiamo scelto: Cozzo Sannita, Monte Presepio, Pizzo Pipitone, Il Pizzo di Ciminna, Rocca di Sciara; Balza Falcone; Monte Piombino; Polizzi Generosa; Serra di Puccia; Cozzo Puccia; Terravecchia di Cuti; Monte Chibbò; Serre del Monaco e abbiamo aggiunto anche 
l'insediamento di Colle Madore (Vassallo, 1999) per l'importanza che doveva avere assunto nel territorio anche se non rientra tra quelli esaminati nel nostro campione.

Il file raster ottenuto è stato utilizzato nel prosieguo della nostra ricerca come filtro nell'individuazione delle aree a "rischio archeologico" grazie ad analisi di modellistica parametrica (regressione logistica).

La ricerca condotta ha permesso che si realizzassero due modelli per l'individuazione di insediamenti di età greca nel territorio della colonia di Himera: un primo modello ci ha permesso di individuare grandi insediamenti a controllo del territorio, mentre il secondo è stato realizzato al fine di rintracciare i piccoli insediamenti a vocazione agricola, tenendo conto della sostanziale differenza nella tipologia insediamentale che doveva esistere in antico, per cui le caratteristiche dei piccoli centri rurali e dell'insediamento disperso sono molto diverse rispetto a quelle dei centri principali con precise funzioni simboliche, politiche e militari. Rimandando ad altra sede i riscontri della ricerca e l'analisi specifica dei due modelli, in questo momento ci interessa valutare come in entrambi i modelli l'Intervisibilità abbia un ruolo fondamentale nelle scelte insediamentali essendo per il primo modello il parametro che più influenza l'individuazione di insediamenti. Nel secondo modello atto all'individuazione di piccoli insediamenti rurali, invece, il parametro dell'intervisibilità è al terzo posto tra i fattori che avrebbero influenzato le scelte insediamentali dopo la pedologia e la distanza dalla viabilità antica.

\section{Bibliografia}

Attema P, GJ Burgers, E Van Joolen, M Van Leusen \& B Mater 2002: New developments in Italian landscape archaeology: theory and methodology of field survey, land evaluation and landscape perception, pottery production and distribution: proceedings of a threeday conference held at the University of Groningen,
April 13-15, 2000, Archaeopress, Oxford.

Belvedere 0, 2010: Contatto culturale e interrelazioni tra Greci e Indigeni nel territorio di Himera, in Tréziny $\mathrm{H}$ (ed.), Grecs et indigènes de la Catalogne à la Mer Noire: actes des rencontres du programme européen Ramses 2 (2006-2008): 55-62. Errance, Paris.

Belvedere 0, A Bertini, G Boschian, A Burgio, A Contino, RM Cucco \& D Lauro (ed.) 2002: Himera 3.2. Prospezione archeologica nel territorio, L'Erma di Bretschneider, Roma.

Burgio A, 2002: Resuttano (IGM 260 III SO), L. S. Olschki, Firenze.

Clarke D, 1977: Spatial archaeology, Academic Press, Boston.

Cucco RM, 1999-2000: Ricognizione archeologica nella valle del F. Torto: avvio di un progetto per la realizzazione di un Sistema Informativo Territoriale storico-archeologico, Dottorato di ricerca in Metodologie conoscitive per la conservazione e la valorizzazione dei Beni Culturali (XII ciclo), Seconda Università di Napoli, S. Maria Capua Vetere.

Curti E, 2003: Il Pizzo di Ciminna, in Atti del convegno “Dal Sikanicon all'Ellenikon. Riflessioni sugli ethne della Sicilia antica. Origini e relazioni”, Regione Siciliana, Palermo.

Di Leonardo L, 2007: II Pizzo, in Spatafora F \& Vassallo $S$ (ed.), Memorie dalla terra. Insediamenti ellenistici nelle vallate della Sicilia Centro-Settentrionale : Caltavuturo, Museo civico chiostro San Francesco, 19 ottobre -2 dicembre 2007: 65-67. Regione Siciliana, Palermo.

Epifanio E, 1980: Terravecchia di Cuti. Scavi e ricerche negli anni 1977-1979, in Beni Culturali e Ambientali Sicilia, 1, 105-108.

Epifanio E, 1993: Terravecchia di Cuti, in Di Terra in Terra. Nuove scoperte archeologiche nella provincia di Palermo. Catalogo della Mostra: 44-48. Regione Siciliana, Palermo.

Fiorani D, 2004-2005: Prospezione archeologica della valle del Torrente Caltavuturo (IGM F 259 I NE Caltavuturo), tesi di laurea, Università degli Studi di Palermo.

Francovich R, H Patterson \& G Barker (ed.) 2000: Extracting meaning from ploughsoil assemblages, Oxbow books, Oxford. 
Lauro D, 2010: Sambuchi (IGM 259 IV SE), L. S. Olschki, Firenze.

Papa MA 2012: Analisi spaziale e modellistica parametrica per la ricerca delle dinamiche insediative

nel comprensorio territoriale e storico-culturale dell'antica colonia d'Himera, tesi di dottorato, Seconda Università degli studi di Napoli (XXIII Ciclo).

Papa MA, 2014: Analisi spaziale e modellistica parametrica per la ricerca delle dinamiche insediative nel comprensorio territoriale e storicoculturale dell'antica colonia di Himera, in Atti del Convegno XVIII CIAC: Centro y periferia en el Mundo Clásico, S. 2. La Arqueología del paisaje. Los recursos naturales, pp. 139-142, Merida.

Vassallo S, 1990: S. Caterina Villarmosa, L. S. Olschki, Firenze.

Vassallo S, 1996: Pizzo di Ciminna, in Bibliografia Topografica della Colonizzazione Greca, vol. 14: 3032. M. D’Auria Editore, Pisa/Roma/Napoli.

Vassallo S, 1999: Colle Madore. Un caso di ellenizzazione in terra sicana, Regione Siciliana, Palermo.

Wheatley D \& M Gillings 2002: Spatial technology and archaeology. The archaeological applications of GIS, Taylor \& Francis, London/New York. 
